\title{
Bayesian calibration of the Schwartz-Smith Model adapted to the energy market
}

\author{
Saikat Saha
}

\section{Linköping University Post Print}

\section{Tweet}

N.B.: When citing this work, cite the original article.

(C2014 IEEE. Personal use of this material is permitted. However, permission to reprint/republish this material for advertising or promotional purposes or for creating new collective works for resale or redistribution to servers or lists, or to reuse any copyrighted component of this work in other works must be obtained from the IEEE.

Saikat Saha, Bayesian calibration of the Schwartz-Smith Model adapted to the energy market, 2014, IEEE statistical signal processing workshop (SSP 2014), Gold Coast, Australia. 


\title{
BAYESIAN CALIBRATION OF THE SCHWARTZ-SMITH MODEL ADAPTED TO THE ENERGY MARKET
}

\author{
Saikat Saha* \\ Division of Automatic Control, Linköping University, Sweden \\ sahadisy. liu.se
}

\begin{abstract}
We consider an application of Bayesian signal processing to the energy trading problem. In particular, we address the problem of calibrating the Schwartz-Smith Model using the observed electricity futures prices traded on the markets. As compared with the other financial markets, basic electricity derivatives such as futures are more complicated, as these products are based not on the spot prices themselves but on the arithmetic averages of the spot prices during the delivery period. As a result, the (log) futures prices are no longer affine function of the model factors and as such, an approach based on Kalman filtering, to estimate the latent model factors and the parameters seems meaningless. Here, we envisage a Bayesian approach using the particle marginal Metropolis Hastings (PMMH) algorithm for this challenging estimation task. We demonstrate the efficacy of our approach on simulated data.
\end{abstract}

Index Terms - PMMH, PMCMC, SMC, SchwartzSmith model, financial signal processing

\section{INTRODUCTION}

Schwartz-Smith two factor model [1] is very popular in practice for modelling commodity prices. In this approach, the spot dynamics is explicitly defined in terms of hidden factors, from which the forward dynamics can be constructed. The popularity of this model partially stems from the fact that the solution for futures prices can be obtained analytically. However, one key issue is calibrating the model against the unknown parameters [2],[3]. The estimation problem becomes difficult as the factors of this model are not directly observable and the futures prices (observations) themselves contain some observation noises attributed to the lack of liquidity and/or high bid ask spread [4]. As shown in [1], the log of the futures prices in this model are affine in the unobserved factors. As a result, Kalman filter and the maximum likelihood method, for example, can be used to estimate the parameters and the unobserved factors from the observed futures data.

The huge success of this model has led to its adaption in the energy market as well. However, unlike the standard fu-

\footnotetext{
*The author would like to thank COOP-LOC, funded by SSF and CADICS, funded by Swedish Research Council (VR) for the financial supports.
}

tures such as the futures on commodity, electricity futures are bit different in the sense that the futures have delivery over a period $\left[T_{0}, T\right]$, rather than at a specific time $T$. Here the payoff structure depends on the arithmetic average of the spot prices over the delivery period [5]. As the sum of the lognormal random variables is not lognormal, consequently, the $\log$ futures are no longer affine function of the hidden factors. In the literature, often for simplicity, the arithmetic average is approximated by a geometric average, so that a tractable affine structure is preserved and the standard Kalman filter can be used [6]. To avoid this ad-hoc approach, recently particle filter (PF) based Bayesian parameter estimation method has been proposed [7]. However, unlike the point estimation (using particle based maximum likelihood or EM [8, 9]), Bayesian parameter estimation with PF has largely remained an unresolved issue and Markov chain Monte Carlo (MCMC) still appears to be the main workhorse for the Bayesian parameter inference.

Particle MCMC (PMCMC) has recently been envisaged as a class of MCMC methods to the dynamic state space model[10]. PMCMC uses sequential Monte Carlo (SMC) aka particle filter, as a proposal mechanism for the state sequence within the MCMC. In this article, we focus solely on the challenging parameter inference problem for the modified Schwartz-Smith model and consider a specific PMCMC algorithm, known as particle marginal Metropolis Hastings (PMMH) for the job. To the best of our knowledge, this has not been considered in earlier studies.

The organization of the article is as follows. Section 2 provides a brief overview of the Schwartz-Smith Model, followed by a modification for extended delivery period in Section 3. The state space representation of the (modified) model is provided in Section 4. We then provide brief descriptions of PMMH algorithm for parameter inference in Section 5. The numerical simulations in Section 6 followed by the conclusions.

\section{SCHWARTZ-SMITH MODEL}

We consider a complete probability space $(\Omega, \mathcal{F}, \mathbb{P})$ with a filtration $\mathbb{F}=\left(\mathcal{F}_{t}\right)_{t \geq 0}$, where $\mathcal{F}_{t}$ represents the information available at time $t$. We use the notation $\mathbf{E}_{t}[X(T)]$ to denote the conditional expectation $\mathbf{E}\left(X(T) \mid \mathcal{F}_{t}\right)$.

Let $S(t)$ represent the spot price of a commodity at time 
$t$. As in [1], we decompose the logarithm of the spot price into two stochastic factors as

$$
\ln (S(t))=\chi(t)+\xi(t)+h(t)
$$

where $h(t)$ is a deterministic seasonality function. $\chi(t)$ and $\xi(t)$ represent the short-term price deviation and the equilibrium price level, following

$$
\begin{aligned}
d \chi(t) & =-\kappa \chi(t) d t+\sigma_{\chi} d W_{\chi}(t) \\
d \xi(t) & =\mu_{\xi} d t+\sigma_{\xi} d W_{\xi}(t) .
\end{aligned}
$$

Here $W_{\chi}$ and $W_{\xi}$ are correlated standard Wiener processes under the real world measure $\mathbb{P}$, with $d W_{\chi}(t) d W_{\xi}(t)=\rho d t$. For the valuation of the futures, we need to represent the model under the risk neutral measure, denoted by $\mathbb{Q}$. Setting technicalities aside, one can show that the risk neutral stochastic process for the two factors are of the form [1]:

$$
\begin{aligned}
& d \chi(t)=\left(-\kappa \chi(t)-\lambda_{\chi}\right) d t+\sigma_{\chi} d W_{\chi}^{*}(t) \\
& d \xi(t)=\left(\mu_{\xi}-\lambda_{\xi}\right) d t+\sigma_{\xi} d W_{\xi}^{*}(t)
\end{aligned}
$$

where $W_{\chi}^{*}$ and $W_{\xi}^{*}$ are correlated Wiener processes under the risk neutral measure, with $d W_{\chi}^{*} d W_{\xi}^{*}=\rho d t$. We denote the current time by $t$, the maturity of the futures by $T$, the time to maturity by $\tau$ where $\tau=T-t$, and $T^{*}$ is a fixed time horizon such that $t \leq T<T^{*}$. The logarithm of the futures price, $\ln F(t, T)$ can be shown to be given by

$$
\ln F(t, T)=e^{-\kappa(T-t)} \chi(t)+\xi(t)+A(t, T),
$$

where

$$
\begin{aligned}
A(t, T) & =\frac{\lambda_{\chi}}{\kappa}\left(e^{-\kappa(T-t)}-1\right)+\left(\mu_{\xi}-\lambda_{\xi}\right)(T-t)+ \\
& +\frac{1}{2}\left[\frac{\sigma_{\chi}^{2}}{2 \kappa}\left(1-e^{-2 \kappa(T-t)}\right)+\sigma_{\xi}^{2}(T-t)+\right. \\
& \left.+\frac{2 \rho \sigma_{\chi} \sigma_{\xi}}{\kappa}\left(1-e^{-\kappa(T-t)}\right)\right]+h(T) .
\end{aligned}
$$

\section{MODIFICATION FOR EXTENDED DELIVERY PERIOD}

The market prices of electricity futures are different from the standard futures such as futures on commodities, as these prices are based on the arithmetic averages of the spot prices over a delivery period: $\frac{1}{n} \sum_{i=1}^{n} S\left(t_{d_{i}}\right) ;\left\{t_{d_{i}}\right\}_{i=1}^{n} \in\left[T_{0}, T\right]$, where $n$ is the number of days in the delivery period $\left[T_{0}, T\right]$. Now, for $t<T$, the futures price can be given by

$$
F\left(t, T_{0}, T\right)=\mathbf{E}\left\{\frac{1}{n} \sum_{i=1}^{n} S\left(t_{d_{i}} \mid \mathcal{F}_{t}\right)\right\}
$$

where $\mathcal{F}_{t}=\sigma\{S(\eta) ; 0 \leq \eta \leq t\}$. Now using the linearity of the expectation operator and the definition of futures price, (7) can be written as

$$
F\left(t, T_{0}, T\right)=\frac{1}{n} \sum_{i=1}^{n} F\left(t, t_{d_{i}}\right) .
$$

Hence from (5) we have,

$$
F\left(t, T_{0}, T\right)=\frac{1}{n} \sum_{i=1}^{n} e^{\left[e^{-\kappa\left(t_{d_{i}}-t\right)} \chi(t)+\xi(t)+A\left(t, t_{d_{i}}\right)\right]} .
$$

\section{STATE SPACE MODEL}

As shown in the original article [1], the state transition dynamics is given by

$$
x_{k+1}=A_{k} x_{k}+U_{k}+W_{k}, \quad k=1, \cdots, N
$$

where

$$
\begin{gathered}
x_{k}=\left[\begin{array}{l}
\chi\left(t_{k}\right) \\
\xi\left(t_{k}\right)
\end{array}\right], A_{k}=\left[\begin{array}{cc}
e^{-\kappa \Delta t} & 0 \\
0 & 1
\end{array}\right] \\
U_{k}=\left[\begin{array}{c}
-e^{-\kappa \Delta t} \lambda_{\chi} \Delta t \\
\left(\mu_{\xi}-\lambda_{\xi}\right) \Delta t
\end{array}\right], W_{k}=\left[\begin{array}{c}
\Delta W_{\chi}\left(t_{k}\right) \\
\Delta W_{\xi}\left(t_{k}\right)
\end{array}\right]
\end{gathered}
$$

with

$$
\begin{gathered}
\Delta W_{k} \sim \mathcal{N}\left(m^{s}, \Sigma_{s}\right) \\
m^{s}=\mathbf{E}\left(\Delta W_{k}\right)^{\prime}=\left[\begin{array}{ll}
0 & 0
\end{array}\right]^{\prime} \\
\Sigma_{s}=\operatorname{Cov}\left(\Delta W_{k}\right)=\mathbf{E}\left(\Delta W_{k} \Delta W_{k}^{\prime}\right) \\
=\left[\begin{array}{cc}
\left(1-e^{-2 \kappa \Delta t}\right) \frac{\sigma_{\chi}^{2}}{2 \kappa} & \left(1-e^{-\kappa \Delta t}\right) \frac{\rho \sigma_{\chi} \sigma_{\xi}}{\kappa} \\
\left(1-e^{-\kappa \Delta t}\right) \frac{\rho \sigma_{\chi} \sigma_{\xi}}{\kappa} & \sigma_{\xi}^{2} \Delta t
\end{array}\right] \text { 13) }
\end{gathered}
$$

where $N$ is the number of time periods in the data set and $\Delta t=t_{k}-t_{k-1}$.

For the measurement equation, the observation data is available on daily basis. We use a sequence of $m$ futures for each time step. For each futures $j=1, \cdots m$, we set the starting time to delivery, $\tau_{j}=T_{0}^{j}-t$ for each current time as constant. Moreover, the delivery period $T-T_{0}=\theta$ is set as a constant (1-month) for all the futures. So, for futures $j$ at time step $t_{i}$, (9) can be written as

$F_{j}\left(t_{i}, T_{0}^{j}, T^{j}\right)=\frac{1}{n} \sum_{i=1}^{n} e^{\left[e^{-\kappa\left(t_{d_{i}}^{j}-t_{i}\right)} \chi\left(t_{i}\right)+\xi\left(t_{i}\right)+A\left(t_{i}, t_{d_{i}}^{j}\right)\right]}$.

As said before, the observed future prices contain some observation noises attributed to the lack of liquidity and/or high bid ask spread. As a result, the following observation model is considered:

$y_{j}\left(t_{i}\right)=\ln \left[\frac{1}{n} \sum_{i=1}^{n} e^{\left[e^{-\kappa\left(t_{d_{i}}^{j}-t_{i}\right)} \chi\left(t_{i}\right)+\xi\left(t_{i}\right)+A\left(t_{i}, t_{d_{i}}^{j}\right)\right]}\right]+\epsilon_{i j}$

where the measurement noise $\epsilon_{i j} \sim \mathcal{N}(0, R)$. For simplicity, $R$ is assumed to be diagonal such that $R(j, j)=s_{j}^{2}$. Further we assume $p\left(x_{0}\right)$ to be known, which is individually uncorrelated to the process and measurement noises respectively. 


\section{BAYESIAN INFERENCE ON THE PARAMETER USING PMMH ALGORITHM}

Consider the problem of identifying the unknown parameter $\theta \in \Theta$ for the discrete time state space model given by

$$
\begin{aligned}
x_{t+1} & \sim p_{\theta}\left(x_{t+1} \mid x_{t}\right) \\
y_{t} & \sim p_{\theta}\left(y_{t} \mid x_{t}\right),
\end{aligned}
$$

where the latent state $x_{t} \in \mathcal{X}$ is following a Markov process, fully characterized by the the initial density $\mu_{\theta}(\cdot)$ and the transitional kernel $p_{\theta}\left(x_{t} \mid x_{t-1}\right)$. The observations $\left\{y_{t}\right\} \in \mathcal{Y}$ are conditionally independent given $\left\{x_{t}\right\}$ and are characterized by the observation kernel $p_{\theta}\left(y_{t} \mid x_{t}\right)$.

Now having the observations $y_{1: T} \triangleq\left(y_{1}, y_{2}, \cdots, y_{T}\right)$, our objective is to perform a Bayesian inference on the parameter $\theta$. For this purpose, we model $\theta$ as a random variable with prior density $\pi(\theta)$. The target posterior of $\theta$ can then be obtained as

$$
p\left(\theta \mid y_{1: T}\right) \propto p\left(y_{1: T} \mid \theta\right) \pi(\theta) .
$$

In the Bayesian formalism, one standard way of sampling from this posterior is obtained through a popular class of algorithms known as MCMC. Metropolis Hastings (MH) is a generic algorithm within this class, where a set of samples $\left\{\theta_{j}\right\}_{j=1}^{J}$ from the posterior is generated using two steps:

1. first, a new sample $\theta^{\prime}$ is drawn according to a chosen proposal distribution $q\left(\cdot \mid \theta_{j-1}\right)$

2. then the sample $\left(\theta_{j}=\theta^{\prime}\right)$ is accepted with probability

$$
\alpha\left(\theta^{\prime}, \theta\right)=\min \left\{1, \frac{\pi\left(\theta^{\prime}\right) p\left(y_{1: T} \mid \theta^{\prime}\right) q\left(\theta_{j-1} \mid \theta^{\prime}\right)}{\pi\left(\theta_{j-1}\right) p\left(y_{1: T} \mid \theta_{j-1}\right) q\left(\theta^{\prime} \mid \theta_{j-1}\right)}\right\} .
$$

The acceptance probability thus depends on the likelihood $p\left(y_{1: T} \mid \theta\right)$, which is, in general, intractable for the model specified by (16a)-(16b). However, the above MCMC sampler can still target the correct posterior, provided the likelihood is replaced with an unbiased estimate: an important result established in [11].

Here we specifically envisage PMMH algorithm, which uses a sequential Monte Carlo (SMC) sampler to compute an unbiased estimate of the likelihood[10]. SMC sampler are a class of simulation methods for approximating a sequence of target distributions in the form of weighted samples[12]. For the state space model in (16a)-(16b), using SMC sampler, the posterior distribution, e.g., $p_{\theta}\left(x_{t} \mid y_{1: t}\right)$, can be approximated by an empirical distribution formed by the weighted samples (particles) as $p_{\theta}\left(x_{t} \mid y_{1: t}\right) \approx \sum_{i=1}^{N} \omega_{t}^{(i)} \delta_{x_{t}^{(i)}}\left(d x_{t}\right)$. Here $\omega_{t}^{(i)}$ is the weight associated with the sample $x_{t}^{(i)}$ and $\delta(\cdot)$ is the Dirac delta function. Within this setup, the estimate of the likelihood $p\left(y_{1: T} \mid \theta\right)$ is then obtained as

$$
\hat{p}\left(y_{1: T} \mid \theta\right)=\prod_{t=1}^{T}\left(\frac{1}{N} \sum_{i=1}^{N} \omega_{t}^{(i)}\right),
$$

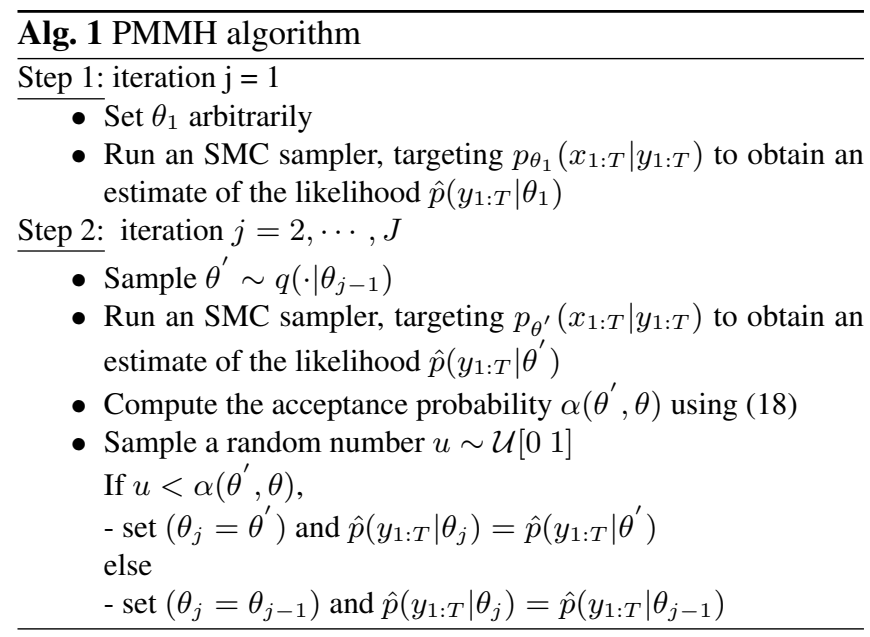

which is shown to be unbiased in [13]. This is an elegant approach with strong theoretical supports, yet the implementation is very simple. For lack of space, we do not discuss it further and instead, only provide an outline of PMMH algorithm (Alg. 1) for our parameter estimation problem. We refer the interested readers to the original article [10] for the details.

\section{SIMULATION STUDY}

We apply our proposed approach on the observation data consist of simulated futures prices with maturity starting in two months (60 days) and constant delivery periods of one month (30 days). The futures price consists of $T=100$ time points with $\Delta t=1$. We do not consider any seasonality function as data are generated synthetically using (10)-(15). The unknown parameters are $\theta=\left\{\kappa, \lambda_{\chi}, \lambda_{\xi}, \sigma_{\chi}, \sigma_{\xi}, \mu_{\xi}, \rho, R\right\}$. For the simulated data, the true (representative) values of the parameters are adapted from [6] and are shown in the Table 1. For simplicity, we assume that the initial state vector is deterministic, given by $x_{0}=\left[\begin{array}{ll}0 & 0\end{array}\right]^{\prime}$. We run the PMMH algorithm with 10000 iterations and a burn-in period of 2000 . For the SMC sampler, we use a simple bootstrap particle filter with 1000 particles. A simple Gaussian random walk proposal was taken for each parameter with variance $=0.05$. All parameters except $\rho$ are assigned the same uninformative inverse Gamma prior. The prior for $\rho$ is taken as the uniform density $U \sim[-1,1]$. The resulting posterior density estimates are shown in Figure 1. This calibration task is

Table 1. Model parameters

\begin{tabular}{c|cccc}
\hline & $\kappa$ & $\lambda_{\chi}$ & $\lambda_{\xi}$ & $\sigma_{\chi}$ \\
\hline True & 1.321 & 0.623 & 0.269 & 0.21 \\
\hline \hline & $\sigma_{\xi}$ & $\mu_{\xi}$ & $\rho$ & $R$ \\
\hline True & 0.07 & 0.296 & 0.588 & 0.01 \\
\hline
\end{tabular}

already known to be a difficult one. However, from Figure 1, 

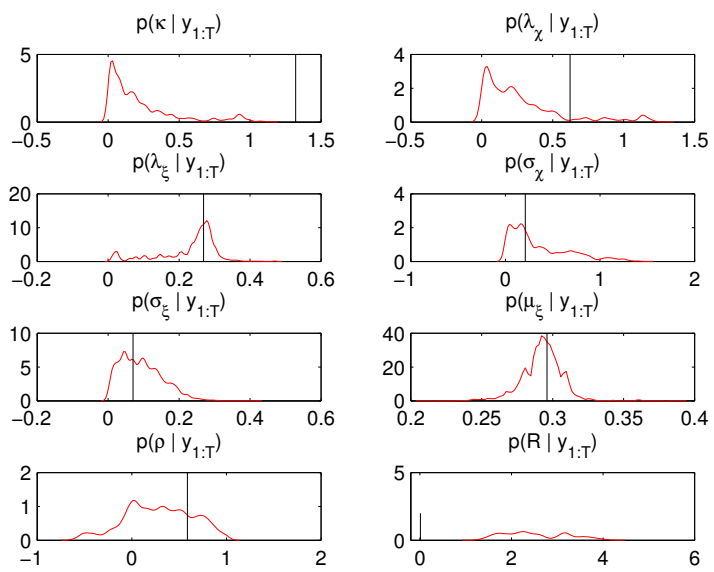

Fig. 1. The posterior density estimates of the parameters with the vertical lines indicating the true parameter values

the estimated posterior densities for the parameters appear to be quite satisfactory. Except for $\kappa$, and $R$, all parameters can be inferred reasonably well.

\section{DISCUSSIONS}

In this article, we address a practical but complex problem of calibrating the Schwartz-Smith model using electricity futures data ${ }^{1}$. We envisage PMMH algorithm, which is relatively new and a very powerful tool, yet quite simple in terms of implementation. We have shown that even with the plain vanilla choice for PMMH, we obtained satisfactory results. There are couple of factors which might have affected the performance here. For example, we have considered very basic SMC sampler, i.e. bootstrap sampler with resampling at each stage. The proposal taken for the bootstrap is not fully adapted and we considered simple multinomial resampling. Together with the moderate number of samples (particles) used here, they might have contributed to the increased variance of the marginal likelihood estimate $\hat{p}\left(y_{1: T} \mid \theta\right)$. This variance can possibly be reduced with a better implementation of SMC sampler.

We have also used the standard random walk proposal for PMMH. As is well known, this proposal does not scale well with the number of parameters; the information from SMC samplers (e.g., score and observed information matrix) can be efficiently incorporated into the proposal design for better performance $[14,15]$. We are currently investigating a new alternative proposal by incorporating the score information within it, with a linear computational cost.

Finally, comparing this PMMH based approach with other

\footnotetext{
${ }^{1}$ In electricity market, jump is a fundamental property. Though our study has not considered the jump case, it can be easily extended.
}

existing methods and using real futures data are left for our future work.

\section{References}

[1] E. Schwartz and J. Smith, "Short-term variations and longterm dynamics in commodity prices," Management Science, vol. 46, no. 7, pp. 893-911, 2000.

[2] T. Perez, G. C. Goodwin, and B. Godoy, "Parameter estimation of structural commodity price models," in Proceedings of SYSID, 2009.

[3] M. Fu and X. Tai, "On parameter estimation of the SchwartzSmith short-term/long-term model," in Proceedings of IEEE ICASSP, 2009.

[4] R. Elliott and C Hyndman, "Parameter estimation in commodity markets: A filtering approach," Journal of Economic Dynamics and Control, vol. 31, pp. 2350-2373, 2007.

[5] F. Benth, J. Benth, and S. Koekebakker, Stochastic modelling of Electricity and Related Markets, World Scientific Publishing, UK, 2008.

[6] M. Kholopova, Estimating a two factor model for the forward rate of electricity, Ph.D. thesis, University of Twente, The Netherlands, 2006.

[7] E. Imreizeeq, Parameter Estimation of a New Energy Spot Model from Futures Prices, Ph.D. thesis, University of Twente, The Netherlands, 2011.

[8] R. Douc, E. Moulines, and D. Stoffer, Nonlinear Time Series: Theory, Methods and Applications with R Examples, Chapman \& Hall/CRC, 2014.

[9] D. Tornqvist, S. Saha, and F. Gustafsson, "Batched Fault Detection using Particle Smoothing," in Proceedings of IEEE Aerospace Conference, Big Sky, Montana, USA, 2011.

[10] C. Andrieu, A. Doucet, and R. Holenstein, "Particle Markov chain Monte Carlo methods," Journal of the Royal Statistical Society: Series B, vol. 72, no. 3, pp. 269-342, 2010.

[11] C. Andrieu and G. O. Roberts, "The pseudo-marginal approach for efficient Monte Carlo computations," The Annals of Statistics, vol. 37, no. 2, pp. 697-725, 2009.

[12] O. Cappé, S. J. Godsill, and E. Moulines, "An overview of existing methods and recent advances in sequential Monte Carlo," Proceedings of IEEE, vol. 95 (5), pp. 899-924, 2007.

[13] P. Del Moral, Feynman-Kac Formulae : Genealogical and Interacting Particle Systems with Applications, Springer Verlog New York, 2004.

[14] J. Dahlin, F. Lindsten, and T. B. Schön, "Particle Metropolis Hastings using Langevin dynamics," in Proceedings of the 38th ICASSP, Vancouver, Canada, 2013, pp. 6308-6312.

[15] C. Nemeth and P. Fearnhead, "Particle Metropolis adjusted Langevin algorithms for state space models," ArXiv: 1402.0694v1, Feb. 2014. 